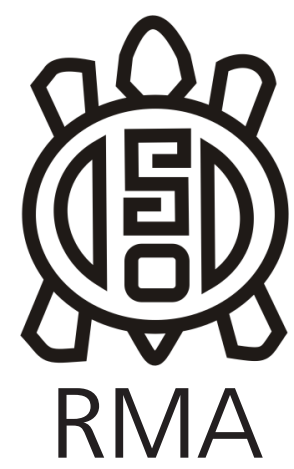

Dossier

\title{
Cambios entesiales en Portugal: un estudio diacrónico utilizando el nuevo Método Coimbra
}

\author{
Entheseal changes in Portugal: a diachronic study applying the new \\ Coimbra Method \\ *Soledad Salega, **Charlotte Henderson, **Ana Maria Silva
}

*IDACOR-CONICET, Museo de Antropología, Facultad de Filosofía y Humanidades, Universidad Nacional de Córdoba, Argentina. E-mail: soledadsalega@gmail.com ${ }^{*}$ CIAS - Centro de Investigação em Antropologia e Saúde, Departamento de Ciencias da Vida, Universidade de Coimbra, Portugal. E-mail: c.y.henderson@uc.pt; amgsilva@uc.pt

\begin{abstract}
Resumen
El objetivo de este trabajo es estudiar las variaciones en la frecuencia de cambios entesiales a través del tiempo en Portugal para evaluar el efecto del aumento de la urbanización y la especialización laboral, aplicando un método de registro biológicamente apropiado, el llamado nuevo método Coimbra (Henderson et al. 2016a). La muestra analizada está compuesta por 138 individuos adultos provenientes de ocho sitios arqueológicos del actual territorio de Portugal, y con cronologías medievales y modernas. Se realizaron cálculos de prevalencias y de odds ratios, así como un test exacto de Fisher para los cambios registrados en once entesis de miembros superiores e inferiores. Los resultados sugieren que la actividad es un factor explicativo de los cambios entesiales registrados, pero que asimismo se deben considerar otros factores, ya que los diferentes perfiles de sexo y edad también contribuyen a las distintas tendencias. Además, el tamaño variable de las muestras de cada subgrupo también aporta a las altas frecuencias de algunos cambios. Sin embargo, este enfoque es fundamental para entender las tendencias temporales de los cambios entesiales.
\end{abstract}

Palabras clave: entesis; actividad; nuevo método Coimbra; tendencias temporales; Portugal.

\begin{abstract}
The aim of this work is to study the changes in entheseal change frequency through time in Portugal in order to evaluate the effect of increased urbanisation and the specificity of labour tasks, by applying a biologically appropriate recording method, the new Coimbra method (Henderson et al. 2016a). The analyzed sample included 138 adult individuals from eight archaeological sites from Portugal, dating from medieval and modern times. Prevalences and odds ratios were calculated, and a Fisher exact test was performed for the entheseal changes that were recorded in eleven entheses from upper and lower limbs. Results suggest that activity is one explanatory factor for the entheseal changes, but others should we considered, since different age and sex profiles also contribute to the different trends. Moreover, different sample sizes in each subgroup also contribute to high frequencies of some changes. However, this approach is vital to understand temporal trends in entheseal changes.
\end{abstract}

Keywords: entheses; activity; new Coimbra method; temporal trends; Portugal.

Los cambios entesiales (Jurmain y Villotte 2010) se han utilizado tradicionalmente como indicadores de actividad física, como una forma de reconstruir los modos de vida de las poblaciones del pasado (Larsen 2002). Se han relacionado por ejemplo con la división sexual del trabajo (e.g. al-Oumaoui et al. 2004; Robb 1998) y con los diferentes modos de subsistencia, aunque los resultados han diferido. Así, mientras algunos autores encuentran una mayor prevalencia de cambios entesiales para cazadores-recolectores (Larsen 2002; Lieverse et al. 2013), otros plantean que los niveles de afectación son mayores entre grupos agricultores (Eshed et al. 2004). A partir de un meta-análisis de los trabajos publicados entre 1995 y 2012, se encontró que los grupos agricultores tienen la menor frecuencia de cambios entesiales en el pasado, seguidos por los cazadores-recolectores con mayores valores y por último los que viven en un entorno industrial, quienes muestran las frecuencias más altas (Henderson 2013). Sin embargo, los trabajos publicados que fueron analizados utilizaron el método de Hawkey y Merbs (1995), el cual presenta problemas como no diferenciar entre entesis fibrosas y fibrocartilaginosas y no dar cuenta del efecto de la edad (Henderson et al. 2013). Además, el registro fue realizado por diferentes investigadores, por lo que no se puede descartar la posibilidad de errores interobservador (Davis et al. 2013; Henderson et al. 2013).

Por lo tanto, el presente estudio tiene como objetivo estudiar las variaciones en la frecuencia de cambios 
entesiales a través del tiempo en Portugal para evaluar el efecto del aumento de la urbanización y la especialización laboral. Para reducir el error del registro, una sola persona realizó el relevamiento de las entesis, utilizando un método de registro biológicamente apropiado, el llamado nuevo método Coimbra (Henderson et al. 2016a). Éste se originó a partir del grupo de trabajo establecido en el workshop realizado en Coimbra (Portugal) en 2009 (Santos et al. 2011), con los aportes de metodologías previas (Mariotti et al. 2004, 2007; Villotte 2006) y se aplica para las entesis de tipo fibrocartilaginosas, registrando los distintos rasgos de manera separada en las distintas zonas de cada entesis.

\section{Material y métodos}

La muestra analizada está compuesta por 138 individuos adultos -64 masculinos y 74 femeninos- (Tabla 1) procedentes de 8 sitios del centro del actual territorio de Portugal (Figura 1), con distintas cronologías históricas, y que para este trabajo fueron agrupados en dos grandes períodos. Por un lado, el período Medieval, entre los siglos IV y XIV, comprende los sitios Monte da Nora (Elvas), Rua dos Barcos (Santarém), Capela de Nossa Senhora da Vitória (São Jorge, Batalha) y Convento São Francisco (Santarém), los cuales se asocian a contextos medievales (Marques y Silva 2006; Silva 1999a, 1999b). Y por otro lado, el período Moderno, entre los siglos XV y XVI, que incluye a los sitios Largo Cândido dos Reis (Santarém), Cemitério junto a Rua Joaquim António de Aguiar (Sousa Bastos, Coimbra), Rabaçal (Coimbra) y Castelo de Alcácer do Sal. Para este momento, se extienden las urbanizaciones y se difunde la especialización laboral entre los oficios (Cunha y Silva 1994; Silva y Codinha 2002; Silva et al. 2001; Tereso 2009).

La determinación del sexo se realizó de acuerdo con los procedimientos sugeridos por Buikstra y Ubelaker (1994). Se tuvieron en cuenta las características morfológicas de la pelvis (arco ventral, concavidad subpúbica, rama isquiopúbica, escotadura ciática mayor,

Tabla 1. Composición de la muestra analizada.

Table 1. Composition of the analyzed sample.

\begin{tabular}{lcccc}
\hline $\begin{array}{l}\text { Agrupamiento cronológico } \\
\text { ISitio }\end{array}$ & $\begin{array}{c}\text { Masculinos } \\
\text { adultos } \\
\text { jóvenes }\end{array}$ & $\begin{array}{c}\text { Masculinos } \\
\text { adultos } \\
\text { medios/mayores }\end{array}$ & $\begin{array}{c}\text { Femeninos } \\
\text { adultos } \\
\text { jóvenes }\end{array}$ & $\begin{array}{c}\text { Femeninos } \\
\text { adultos } \\
\text { medios/mayores }\end{array}$ \\
$\begin{array}{l}\text { Período Medieval } \\
\text { Monte da Nora }\end{array}$ & 0 & 1 & 0 & 6 \\
Rua dos Barcos & 13 & 17 & 15 & 14 \\
São Jorge-Batalha & 0 & 4 & 0 & 2 \\
Convento São Francisco & 0 & 14 & 5 & 14 \\
\hline Total & 13 & 36 & 20 & 36 \\
\hline Período Moderno & 0 & 11 & 1 & 7 \\
Largo Candido dos Reis & 1 & 2 & 3 & 4 \\
Sousa Bastos & 0 & 0 & 0 & 1 \\
Rabaçal & 0 & 1 & 0 & 2 \\
Alcacer do Sal-Castelo & 1 & 14 & 4 & 14 \\
\hline Total & & & & \\
\hline
\end{tabular}

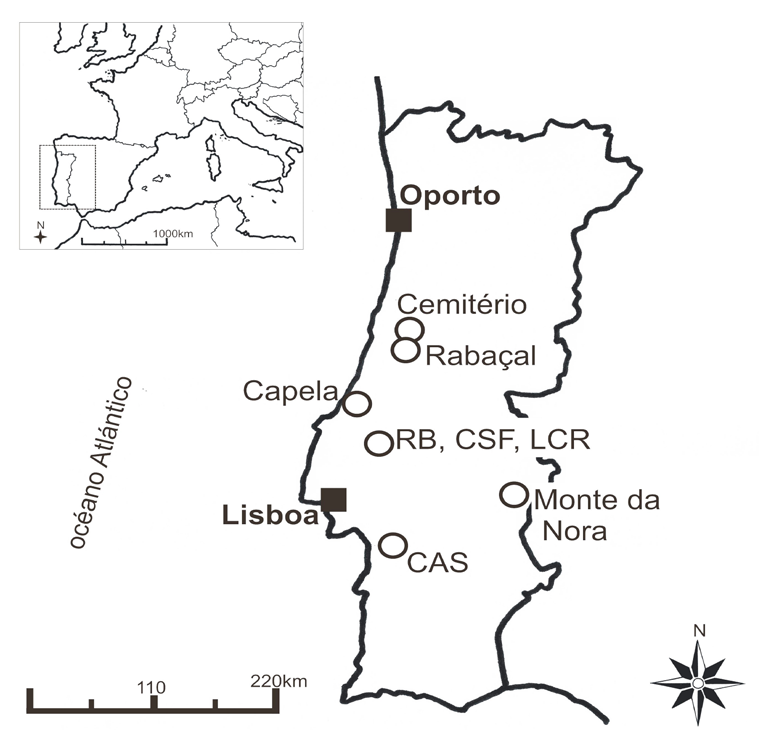

Figura 1. Ubicación de los sitios arqueológicos (puntos blancos). Cemitério $=$ Cemitério junto a Rua Joaquim António de Aguiar; Capela= Capela de Nossa Senhora da Vitória; $\mathrm{RB}=$ Rua dos Barcos; $\mathrm{CSF}=$ Convento São Francisco; LCR= Largo Cândido dos Reis; CAS= Castelo de Alcácer do Sal.

Figure 1. Location of archaeological sites (white points). Cemitério= Cemitério junto a Rua Joaquim António de Aguiar; Capela= Capela de Nossa Senhora da Vitória; $R B=$ Rua dos Barcos; CSF= Convento São Francisco; $L C R=$ Largo Cândido dos Reis; $C A S=$ Castelo de Alcácer do Sal.

surco preauricular) y el cráneo (cresta nucal, proceso mastoideo, margen supraorbital, glabela, eminencia mental). Además, como método complementario, se siguió la propuesta de Albanese (2013) debido a que no todos los esqueletos presentaban las partes anatómicas diagnósticas necesarias para los primeros métodos. En este caso, se consideró el diámetro sagital del radio. Para la estimación de edad, se tuvo en cuenta la morfología del pubis (Brooks y Suchey 1990), la superficie auricular del ilion (Lovejoy et al. 1985) y el grado de fusión de la clavícula (Ubelaker 1987), ya que su extremo esternal completa la fusión alrededor de los 30 años de edad (MacLaughlin 1990), por lo que resultó de utilidad para distinguir entre adultos jóvenes y medios/mayores. En la primera categoría se agruparon los individuos de entre 18 y 30 años de edad, y en la segunda categoría, aquellos mayores de 30 años.

En cuanto al registro de los cambios entesiales, se utilizó el nuevo método Coimbra (Henderson et al. 2016a). En cada entesis se distinguen dos zonas (denominadas zona 1 y zona 2), y en cada una de ellas se registran diferentes rasgos. En la zona 1, la porción más fibrosa de la entesis, se considera la formación ósea y la erosión. En la zona 2, se registran los mismos rasgos (Figura 2) y se suma el cambio textural, la porosidad fina, la macroporosidad y las cavitaciones. Se 


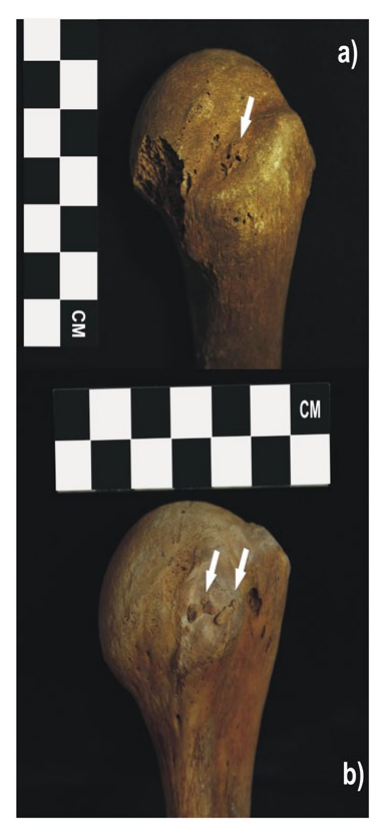

Figura 2. Ejemplos de cambios entesiales: a) erosión en la Zona 2 de la inserción del infraespinal; b) formación ósea en la Zona 2 de la inserción del subescapular.

Figure 2. Examples of entheseal changes: a) erosion in Zone 2 of the infraspinatus insertion; $b$ ) bone formation in Zone 2 of the subscapularis insertion.

utiliza una escala de 0 a 2, con excepción del cambio textural, para el que sólo se considera 0 y 1 . Se seleccionaron once entesis correspondientes a los músculos supraespinoso, infraespinoso, subescapular, extensor común de los dedos, flexor común de los dedos, bíceps braquial, tríceps braquial, semimembranoso (incluyendo semitendinoso), glúteo medio, tendón patelar y tríceps sural, y el registro fue realizado por un solo observador. Las muestras se agruparon de acuerdo con el sexo, la edad (adultos jóvenes y adultos medios/mayores), y la cronología (Medievales y Modernos), teniendo en cuenta los lados izquierdo y derecho en conjunto. En este caso, no se evaluó la lateralidad ya que previamente no se registraron diferencias significativas (Henderson et al. 2017).

Se llevaron a cabo tests de error intra e interobservador calculando porcentajes de acuerdo. Para ello se tomaron 20 individuos, cuyas entesis fueron registradas dos veces por la responsable del registro (SS), con un intervalo de 10 días entre las observaciones, y una vez por la instructora en el método $(\mathrm{CH})$. Este último fue comparado con el segundo registro de SS (ya que éste se encuentra más relacionado con la totalidad del registro, y por lo tanto, con el nivel de acuerdo general). Posteriormente, se realizó un cálculo de frecuencias para cada uno de los rasgos y grados registrados en cada entesis, y finalmente un análisis estadístico de razón de probabilidades $\mathrm{u}$ odds ratios para comparar presencia (grados 1 y 2) versus ausencia (grado 0) de los rasgos. Esta elección responde a que previamente se ha detectado una

Tabla 2. Error intra e interobservador (porcentajes de acuerdo). $F O=$ formación ósea; $E R=$ erosión; $\mathrm{CT}=$ cambio textural; $\mathrm{POF}=$ porosidad fina; $\mathrm{MPO}=$ macroporosidad; $\mathrm{CA}=$ cavitaciones.

Table 2. Intra and interobserver error (percentage agreement). $F O=$ bone formation; $E R=$ erosion; $C T=$ textural change; $P O F=$ fine porosity; $M P O=$ macroporosity; $C A=$ cavitations. baja frecuencia del grado 2, por lo que esto dificulta su inclusión en el análisis estadístico (Henderson et al. 2013). Para el cálculo de los valores estadísticamente significativos se utilizó el test exacto de Fisher, con un nivel de significación de $p \leq 0,05$.

\section{Resultados}

\section{Error intra e interobservador}

Los porcentajes de acuerdo para el test de error intraobservador son iguales o superiores al $90 \%$ en la mayoría de los casos ( $\mathrm{n} / \mathrm{N}=72,7 \%)$, con un porcentaje de acuerdo mínimo de 75\%. En el test interobservador, el porcentaje de acuerdo es igual o superior al $90 \%$ en una menor cantidad de casos ( $\mathrm{n} / \mathrm{N}=69,7 \%)$, con un porcentaje de acuerdo mínimo de 60\% (Tabla 2).

\section{Cálculo de frecuencias}

Con respecto al cálculo de frecuencias de la presencia de los distintos rasgos en cada entesis, se observa que en el período Medieval los mayores valores corresponden a la formación ósea en ambas zonas y a la erosión en zona 2 (Figura 3). En cuanto a la presencia de macroporosidad y cavitaciones, las frecuencias se encuentran entre 1\% y $7 \%$. Estos rasgos se localizan en todas las entesis de los miembros superiores, excepto en el flexor común de los dedos, y sólo en el semimembranoso para los miembros inferiores. Finalmente, el cambio textural y la porosidad fina sólo se registran en el semimembranoso y el tríceps braquial, respectivamente, ambos con frecuencias de $1 \%$. Estas últimas entesis, además, son las más afectadas por cambios en términos cuantitativos ya que presentan 7 y 6 rasgos respectivamente, del total de 8 considerados. Y en cuanto a los valores de frecuencias,

\begin{tabular}{lccccccccc}
\hline & \multicolumn{2}{c}{ Zona 1} & \multicolumn{2}{c}{ Zona 2} & & & & & \\
Entesis & Todos & FO & ER & CT & FO & ER & POF & MPO & CA \\
\hline Intraobservador & & & & & & & & & \\
Supraespinoso & 90,6 & 85,0 & 85,0 & 95,0 & 87,5 & 92,5 & 95,0 & 95,0 & 90,0 \\
Infraespinoso & 88,8 & 87,5 & 90,0 & 92,5 & 82,5 & 85,0 & 92,5 & 90,0 & 90,0 \\
Subescapular & 87,5 & 87,5 & 90,0 & 90,0 & 85,0 & 80,0 & 90,0 & 87,5 & 90,0 \\
Extensor común de los dedos & 92,5 & 82,5 & 92,5 & 97,5 & 87,5 & 92,5 & 97,5 & 95,0 & 95,0 \\
Flexor común de los dedos & 95,0 & 90,0 & 95,0 & 97,5 & 97,5 & 90,0 & 95,0 & 97,5 & 97,5 \\
Bíceps braquial & 90,0 & 77,0 & 90,0 & 97,5 & 85,0 & 90,0 & 95,0 & 87,5 & 97,5 \\
Tríceps braquial & 90,9 & 92,5 & 92,5 & 97,5 & 77,5 & 87,5 & 92,5 & 90,0 & 97,5 \\
Semimembranoso & 85,9 & 80,0 & 85,0 & 90,0 & 77,5 & 75,0 & 90,0 & 90,0 & 100,0 \\
Glúteo medio & 95,6 & 97,5 & 90,0 & 100,0 & 100,0 & 97,5 & 97,5 & 92,5 & 100,0 \\
Tendón patelar & 99,1 & 97,5 & 97,5 & 100,0 & 97,5 & 100,0 & 100,0 & 100,0 & 100,0 \\
Tríceps sural & 90,0 & 75,0 & 85,0 & 95,0 & 87,5 & 95,0 & 92,5 & 95,0 & 95,0 \\
\hline Interobservador & & & & & & & & & \\
Supraespinoso & 94,1 & 87,5 & 90,0 & 100,0 & 90,0 & 87,5 & 97,5 & 100,0 & 100,0 \\
Infraespinoso & 92,8 & 95,0 & 95,0 & 97,5 & 82,5 & 87,5 & 95,0 & 97,5 & 92,5 \\
Subescapular & 78,1 & 72,5 & 77,5 & 87,5 & 60,0 & 80,0 & 85,0 & 77,5 & 85,0 \\
Extensor común de los dedos & 92,8 & 77,5 & 90,0 & 100,0 & 90,0 & 87,5 & 100,0 & 100,0 & 97,5 \\
Flexor común de los dedos & 91,3 & 90,0 & 90,0 & 95,0 & 80,0 & 90,0 & 95,0 & 95,0 & 95,0 \\
Bíceps braquial & 90,0 & 72,5 & 92,5 & 95,0 & 90,0 & 87,5 & 92,5 & 92,5 & 97,5 \\
Tríceps braquial & 95,0 & 85,0 & 95,0 & 100 & 97,5 & 92,5 & 92,5 & 97,5 & 100,0 \\
Semimembranoso & 84,4 & 75,0 & 82,5 & 92,5 & 70,0 & 80,0 & 95,0 & 97,5 & 92,5 \\
Glúteo medio & 90,3 & 77,5 & 85,0 & 97,5 & 87,5 & 95,0 & 90,0 & 92,5 & 97,5 \\
Tendón patelar & 97,2 & 92,5 & 95,0 & 100,0 & 95,0 & 97,5 & 100,0 & 97,5 & 100,0 \\
Tríceps sural & 87,8 & 65,0 & 85,0 & 92,5 & 92,5 & 90,0 & 92,5 & 92,5 & 92,5 \\
\hline & & & & & & & & &
\end{tabular}


los mayores se encuentran en el tríceps sural y el semimembranoso, seguidos por el subescapular y el extensor común de los dedos.

Para el período Moderno, los mayores valores también corresponden a la formación ósea (zonas 1 y 2) y la erosión en la zona 2. Las frecuencias de los tres rasgos son algo mayores que para el período anterior en el caso

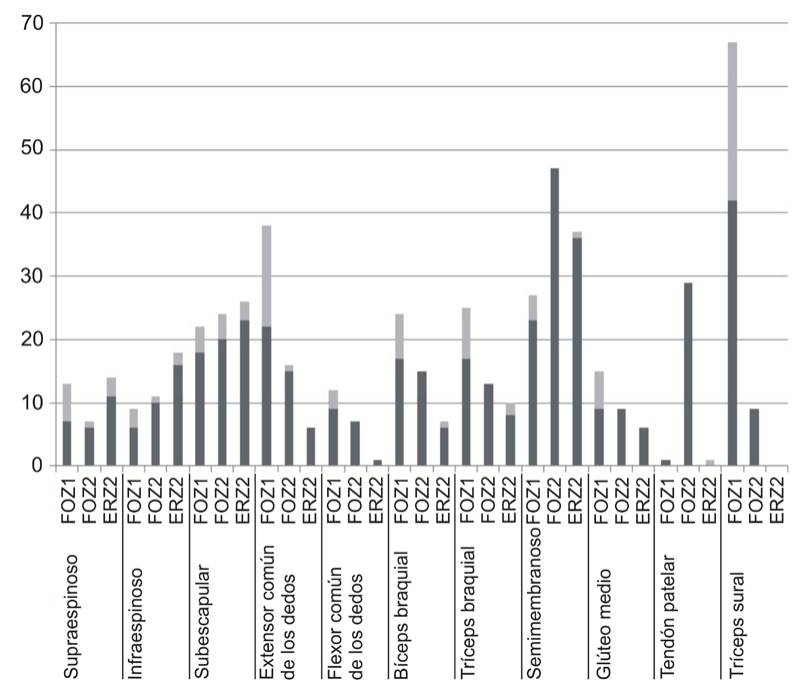

Figura 3. Prevalencias porcentuales en el período Medieval. Sólo se muestran las entesis y los rasgos donde se identificaron las diferencias más marcadas. FOZ1= formación ósea en Zona 1; FOZ2= formación ósea en Zona 2; ERZ2= erosión en Zona 2. El color gris oscuro corresponde al grado 1, y el color gris claro al grado 2 .

Figure 3. Percentage prevalences in the medieval period. Only entheses and features with the most marked differences are shown. FOZ1= bone formation in Zone 1; FOZ2= bone formation in Zone 2; ERZ2= erosion in Zone 2. The dark gray color refers to score 1, and the light gray color to score 2.

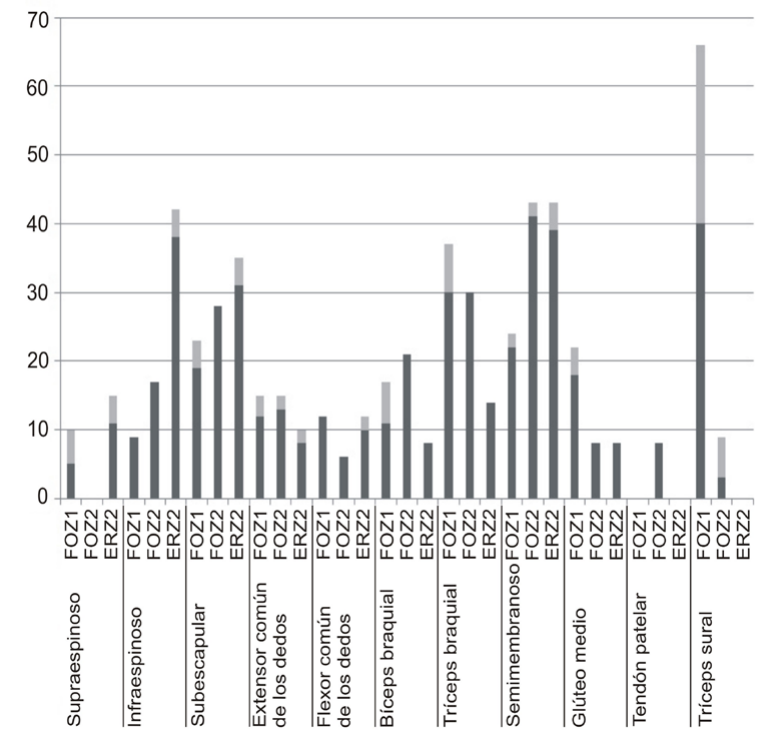

Figura 4. Prevalencias porcentuales en el período Moderno. Ver referencias en epígrafe de Figura 3.

Figure 4. Percentage prevalences in the modern period. See legend for Figure 3. del subescapular, flexor común de los dedos, tríceps braquial y glúteo medio. En el resto de las entesis del miembro superior y el semimembranoso, se presentan valores de frecuencia más altos para la erosión en zona 2 (los correspondientes a formación ósea en ambas zonas se encuentran para el período Medieval) (Figura 4). Los valores correspondientes a cavitaciones y macroporosidad son semejantes al período Medieval, pero hay una menor cantidad de entesis afectadas para el miembro superior (descienden de 7 a 5), mientras que aumentan para el miembro inferior (ascienden de 1 a 3). Nuevamente los rasgos menos representados son el cambio textural y la porosidad fina, aunque ésta última se registra ahora en cinco entesis (subescapular, flexor común de los dedos, bíceps braquial, semimembranoso y glúteo medio). De la misma manera que para el período anterior, las entesis con mayor número de rasgos presentes son el semimembranoso (8) y el bíceps braquial (7), y las mayores frecuencias se registran en el semimembranoso y el tríceps sural, seguidos por el subescapular y el tríceps braquial.

\section{Análisis estadístico}

Si se considera la totalidad de la muestra y se evalúan las diferencias cronológicas, se registran diferencias significativas para el infraespinoso, flexor común de los dedos, tríceps braquial y semimembranoso, con mayores frecuencias para el período Moderno. Si se tiene en cuenta el sexo, no se encuentran diferencias significativas. $Y$ si se tiene en cuenta la edad, los valores más altos se registran en todas las entesis, con excepción del tendón patelar, y corresponden al grupo de los adultos medios/mayores (Tabla 3).

No obstante, cuando se evalúan las diferencias por sexo y edad comparando ambos períodos, se observa que los valores significativos se corresponden casi en su totalidad con las mismas entesis. Para el grupo de individuos femeninos, los valores significativos se encuentran para la formación ósea en zona $1(p=0,016)$ y en zona $2(p=0,003)$ del tríceps braquial (para los individuos masculinos no se registran diferencias significativas). Para el grupo de adultos medios/ mayores, se encuentran para la erosión en zona 2 del flexor común de los dedos $(p=0,003)$, la erosión en zona 1 del tríceps braquial $(p=0,042)$ y el cambio textural del semimembranoso $(p=0,031)$. La excepción está constituida por la formación ósea en zona 2 del tríceps braquial, que también resulta estadísticamente significativa para el grupo de adultos jóvenes $(p=0,019)$. En todos los casos, los valores más altos mencionados corresponden al período Moderno.

\section{Discusión y conclusiones}

En general, los resultados de los tests de error intra e interobservador coinciden con lo encontrado por Wilczak et al. (2017) -para el mismo método- y por 


\begin{tabular}{|c|c|c|c|c|c|c|c|c|c|c|c|}
\hline \multirow{2}{*}{ Entesis } & \multirow{2}{*}{ Rasgo } & \multicolumn{4}{|c|}{ Cronología } & \multirow[b]{2}{*}{$p$} & \multicolumn{5}{|c|}{ Edad } \\
\hline & & & $\mathrm{n} / \mathrm{N}$ & $\%$ & OR & & & $\mathrm{n} / \mathrm{N}$ & $\%$ & OR & $p$ \\
\hline \multirow[t]{2}{*}{ Supraespinoso } & ERZ2 & & & & & & Joven & $1 / 30$ & 3,3 & & \\
\hline & & & & & & & Medio/mayor & $15 / 72$ & 20,8 & 7,632 & 0,020 \\
\hline \multirow[t]{2}{*}{ Infraespinoso } & ERZ2 & Medieval & $14 / 74$ & 18,9 & & & Joven & $2 / 30$ & 6,7 & & \\
\hline & & Moderno & $10 / 24$ & 41,7 & 3,061 & 0,027 & Medio/mayor & $22 / 68$ & 32,3 & 6,696 & 0,004 \\
\hline \multirow[t]{6}{*}{ Subescapular } & FOZ1 & & & & & & Joven & $2 / 31$ & 6,4 & & \\
\hline & & & & & & & Medio/mayor & $20 / 65$ & 30,8 & 6,444 & 0,006 \\
\hline & FOZ2 & & & & & & Joven & $1 / 35$ & 2,8 & & \\
\hline & & & & & & & Medio/mayor & $26 / 71$ & 36,6 & 19,644 & $<0.001$ \\
\hline & ERZ2 & & & & & & Joven & $3 / 35$ & 8,6 & & \\
\hline & & & & & & & Medio/mayor & $27 / 71$ & 38,0 & 6,545 & 0,001 \\
\hline \multirow{4}{*}{$\begin{array}{l}\text { Extensor común } \\
\text { de los dedos }\end{array}$} & FOZ1 & & & & & & Joven & $2 / 41$ & 4,9 & & \\
\hline & & & & & & & Medio/mayor & $46 / 106$ & 43,4 & 14,950 & $<0.001$ \\
\hline & FOZ2 & & & & & & Joven & $2 / 44$ & 4,5 & & \\
\hline & & & & & & & Medio/mayor & $25 / 124$ & 20,2 & 5,303 & 0,010 \\
\hline Flexor común & FOZ1 & & & & & & Joven & $1 / 45$ & 2,2 & & \\
\hline \multirow[t]{3}{*}{ de los dedos } & & & & & & & Medio/mayor & $17 / 106$ & 16,0 & 8,404 & 0,011 \\
\hline & ERZ2 & Medieval & $1 / 140$ & 0,7 & & & & & & & \\
\hline & & Moderno & $6 / 48$ & 12,5 & 19,857 & 0,001 & & & & & \\
\hline \multirow[t]{4}{*}{ Bíceps braquial } & FOZ1 & & & & & & Joven & $6 / 52$ & 11,5 & & \\
\hline & & & & & & & Medio/mayor & $33 / 125$ & 26,4 & 2,750 & 0,021 \\
\hline & FOZ2 & & & & & & Joven & $3 / 53$ & 5,7 & & \\
\hline & & & & & & & Medio/mayor & $27 / 127$ & 21,2 & 4,500 & 0,006 \\
\hline \multirow[t]{6}{*}{ Tríceps braquial } & FOZ1 & & & & & & Joven & $3 / 53$ & 5,7 & & \\
\hline & & & & & & & Medio/mayor & $49 / 133$ & 36,8 & 9,722 & $<0.001$ \\
\hline & FOZ2 & Medieval & $19 / 144$ & 13,2 & & & Joven & $2 / 54$ & 3,7 & & \\
\hline & & Moderno & $13 / 43$ & 30,2 & 2,851 & 0,011 & Medio/mayor & $30 / 133$ & 22,6 & 7,573 & 0,001 \\
\hline & ERZ2 & & & & & & Joven & $2 / 54$ & 3,7 & & \\
\hline & & & & & & & Medio/mayor & $18 / 133$ & 13,5 & 4,070 & 0,036 \\
\hline \multirow[t]{8}{*}{ Semimembranoso } & FOZ1 & & & & & & Joven & $6 / 55$ & 10,9 & & \\
\hline & & & & & & & Medio/mayor & $41 / 123$ & 33,3 & 4,083 & 0,001 \\
\hline & ERZ1 & Medieval & 2/137 & 1,4 & & & & & & & \\
\hline & & Moderno & $4 / 41$ & 9,7 & 7,297 & 0,026 & & & & & \\
\hline & CT & Medieval & $1 / 148$ & 0,7 & & & & & & & \\
\hline & & Moderno & $3 / 44$ & 6,8 & 10,756 & 0,038 & & & & & \\
\hline & FOZ2 & & & & & & Joven & $14 / 58$ & 24,1 & & \\
\hline & & & & & & & Medio/mayor & $74 / 134$ & 55,2 & 3,876 & $<0.001$ \\
\hline \multirow[t]{2}{*}{ Glúteo medio } & FOZ1 & & & & & & Joven & $1 / 34$ & 2,9 & & \\
\hline & & & & & & & Medio/mayor & $16 / 64$ & 25,0 & 11,000 & 0,004 \\
\hline \multirow[t]{2}{*}{ Tríceps sural } & FOZ1 & & & & & & Joven & $3 / 14$ & 21,4 & & \\
\hline & & & & & & & Medio/mayor & $63 / 85$ & 74,1 & 10,500 & $<0.001$ \\
\hline
\end{tabular}

Tabla 3. Valores de odds ratios según cronología, sexo y edad de las muestras. Sólo se presentan los valores estadísticamente significativos $(p \leq 0,05)$. $\mathrm{n} / \mathrm{N}=$ número de cambios registrados/número de entesis observadas; \%= porcentaje de afectación; $\mathrm{OR}=$ odds ratios; $\mathrm{FO}=$ formación ósea; $\mathrm{ER}=$ erosión; $\mathrm{MPO}=$ macroporosidad; $\mathrm{CT}=$ cambio textural; $\mathrm{CA}=$ cavitaciones; $\mathbf{Z 1}=$ zona $1 ; \mathrm{Z} 2=$ zona 2 .

Table 3. Odds ratios values, by chronology, sex, and age of the sample. Only statistically significant values $(p \leq 0.05)$ are presented. $n / N=$ number of recorded changes/number of observable entheses; $\%=$ affected percentage; $O R=$ odds ratios; $F O=$ bone formation; $E R=$ erosion; $M P O=$ macroporosity; $C T=$ textural change; $C A=$ cavitations; $Z 1=$ zone $1 ; Z 2=$ zone 2.

de cambios registrados, lo cual estaría explicando el alto acuerdo logrado entre las observaciones.

En cuanto a la variabilidad de cambios entesiales a través del tiempo, se registró un aumento de éstos en la transición medieval-moderna para la mayoría de las entesis de miembros superiores, y con valores estadísticamente significativos para las del infraespinoso, tríceps braquial y flexor común de los dedos. En los miembros inferiores las frecuencias de los cambios son mayores para los momentos medievales en el caso del tendón patelar y la formación ósea en el resto de las entesis. Si esto se relaciona con las actividades físicas, puede sugerirse que aquellas que afectaron el uso de los miembros superiores se volvieron más exigentes y/o repetitivas. Los cambios registrados serían originados entonces

Mariotti et al. (2007) -para el de su autoría- con respecto a un error cercano al 20\% tanto para los tests intra como interobservador. Una posible explicación es que dichos métodos consideran más de un grado de cambios óseos. No obstante, reducir los grados para así disminuir el error significaría también perder información valiosa (Wilczak et al. 2017). Sin embargo, los valores son variables, por lo que si se considera la media de los acuerdos, éstas muestran una muy buena repetibilidad, tanto para el test interobservador $(90,4)$ como el intraobservador $(91,5)$.

Los porcentajes de acuerdo más altos corresponden a las cavitaciones, la macroporosidad y el cambio textural. Aquí se debe considerar que su presencia en las entesis es menos frecuente que la formación ósea y la erosión. Esto también puede extenderse al tipo de entesis y mencionar, por ejemplo, a la del tendón patelar, cuyos porcentajes de acuerdo son como mínimo 97.5 (intraobservador) y 92.5 (interobservador). No obstante, se trata de la entesis con menor variabilidad por procesos de reparación para reducir el estrés biomecánico, o posiblemente por este mismo estrés (Henderson et al. 2017). Esto además estaría de acuerdo con lo propuesto por Henderson (2013) con respecto a una mayor afectación de poblaciones industriales en comparación con otras de cronologías más antiguas, ya que el tiempo de adaptación a las nuevas condiciones de trabajo y estilo de vida fue reducido. Sin embargo, esto no podría afirmarse de manera segura debido a las limitaciones ya mencionadas al comienzo de este trabajo. Además, el tiempo transcurrido podría no ser lo suficientemente prolongado para que una tendencia evolutiva pueda influir en estos cambios.

Por otra parte, también se registraron valores decrecientes para algunas entesis y rasgos, como en el caso de cavitaciones y macroporosidad en los miembros superiores. Además, a pesar de lo dicho anteriormente sobre las prevalencias en miembros inferiores, se registró un valor significativo para el cambio textural en el semimembranoso en el período Moderno. Si bien 
aumenta la especialización laboral de los oficios y el número de asentamientos urbanos, el modo de vida pudo no haber registrado cambios abruptos (Tereso 2009). Sin embargo, desde el análisis de tendencias seculares de la talla, sí se ha propuesto un desmejoramiento en la calidad de vida para la época moderna, en comparación con períodos previos (Cardoso y Gomes 2008).

Además, la actividad no es el único factor explicativo de los cambios entesiales. En efecto, distintas investigaciones en el campo de la medicina así como de la bioarqueología han demostrado que estos cambios óseos se encuentran relacionados no sólo con la actividad física sino también con factores como la edad, el sexo, la masa corporal y la predisposición genética (Jurmain 2009; Jurmainet al. 2012; Villotte y Knüsel 2013). De todos modos, éstos tampoco pueden extenderse a todos los casos de estudio. Así, por ejemplo, Michopoulou y colaboradores (2016) al aplicar el método Coimbra en una muestra documentada de Atenas muestran que la relación entre cambios entesiales y masa corporal (al menos cuando se utiliza el tamaño corporal como proxy) rara vez resulta estadísticamente significativa.

Asimismo, se debe señalar que los rasgos registrados con el nuevo método Coimbra muestran una variabilidad normal de acuerdo con las entesis en las que se presentan (Henderson et al. 2013, 2017). Por lo tanto, esto podría estar explicando las mayores frecuencias de la macroporosidad y las cavitaciones para los miembros superiores en ambos períodos, o la presencia de cambio textural en la entesis del semimembranoso.

Si se tiene en cuenta el sexo de los individuos, en el presente trabajo no se registraron diferencias significativas al comparar hombres y mujeres. Al dividir la muestra según su cronología, se encontró solo un valor estadísticamente significativo para el grupo de las mujeres del período Moderno, para la formación ósea de ambas áreas del tríceps braquial. Por el contrario, se destaca la cantidad de diferencias significativas entre grupos de edad, al considerar la muestra en su conjunto. Sin embargo, es llamativo que al comparar ambas cronologías separadamente para cada grupo de edad, dicha cantidad se ve reducida y sólo corresponden a tres entesis (tríceps braquial, flexor común de los dedos y semimembranoso). Esto sugiere que la edad no da cuenta de toda la variabilidad de los cambios entesiales. Específicamente para el método utilizado aquí, se ha encontrado que la edad estaría explicando como máximo un $40 \%$ de dicha variabilidad (Henderson et al. 2013, 2016b). Además, el efecto de esta variable es diferente de acuerdo con el rasgo considerado, por ejemplo es más marcado para el desarrollo de la formación ósea (Henderson et al. 2013). En el presente estudio, los valores significativos al evaluar la edad correspondieron a la formación ósea y la erosión.
Una limitación para mencionar refiere a los distintos perfiles de sexo y edad, y los diferentes $n$ de cada uno de los subgrupos en que se dividió la muestra a la hora de realizar comparaciones. Esto contribuye a las distintas tendencias observadas, así como podría estar sobrestimando o subestimando las frecuencias reales de los cambios en las poblaciones. En el caso de los grupos de edad, un mayor $n$ permitiría dividir la categoría medio/mayor, lo cual brindaría mayor detalle en cuanto a los cambios entesiales a lo largo de la vida de los individuos. Por otra parte, en el caso de la división cronológica, el pequeño tamaño muestral para el período Moderno podría estar relacionado con las diferencias identificadas. Sin embargo, este enfoque es fundamental para entender las tendencias temporales de los cambios entesiales y su relación con las modificaciones en los modos de vida y las actividades físicas.

En conclusión, se han encontrado frecuencias de cambios entesiales más altas para el período Moderno con respecto al Medieval. Aunque se sabe que la edad se relaciona con su aparición (como también se vio aquí para los individuos adultos medios/mayores) ésta no es su única causa. Por lo tanto, las distintas frecuencias de cambios probablemente indican algunas diferencias en el estilo de vida causadas por cargas biomecánicas o traumas. No obstante, como se ha mostrado en el presente trabajo, es importante considerar el efecto de la edad y el sexo, así como obtener conclusiones amplias, antes que inferir actividades específicas.

Córdoba y Coimbra, 10 de abril de 2017

\section{Agradecimientos}

Las autoras desean agradecer los comentarios de los evaluadores, los cuales han contribuido a mejorar el presente trabajo.

\section{Bibliografía}

Albanese, J. 2013. A method for estimating sex using the clavicle, humerus, radius, and ulna. Journal of Forensic Sciences, 58 (6): 1413-1419.

al-Oumaoui, I., S. Jiménez Brobeil y P. du Souich. 2004. Markers of activity patterns in some populations of the Iberian Peninsula. International Journal of Osteoarchaeology, 14: 343-359.

Brooks, S. T., J. M. Suchey. 1990. Skeletal age determination based on the os pubis: a comparison of the Acsádi-Nemeskéri and Suchey-Brooks methods. Human Evolution, 5: 227-238.

Buikstra, J., D. Ubelaker (eds.) 1994. Standards for Data Collection from Human Skeletal Remains. Field Museum 
of Natural History, Arkansas Archaeological Survey Research Series, N 44.

Cardoso, H. F. V., J. E. A. Gomes. 2008. Trends in adult stature of peoples who inhabited the modern Portuguese territory from the Mesolithic to the late 20th century. International Journal of Osteoarchaeology, 19 (6): 711-725.

Cunha, E., Silva, A. M. 1994. Intervenção antropológica no Castelo de Alcácer do Sal. Relatórios mensais de Fevereiro a Setembro. Ms.

Davis, C. B., K. A. Shuler, M. E. Danforth y K. E. Herndon. 2013. Patterns of interobserver error in the scoring of entheseal changes. International Journal of Osteoarchaeology, 23: 147-151.

EshedV., A. Gopher, E. Galili e I. Hershkovitz. 2004. Musculoskeletal stress markers in Natufian huntergatherers and Neolithic farmers in the Levant: The upper limb. American Journal of Physical Anthropology, 123: 303-315.

Hawkey, D., C. Merbs. 1995. Activity induced musculoskeletal stress markers (MSM) and subsistence strategy changes among ancient Hudson Bay Eskimos. International Journal of Osteoarchaeology, 5: 324-338.

Henderson, C. Y. 2013. Subsistence strategy changes: the evidence of entheseal changes. Homo - Journal of Comparative Human Biology, 64: 491-508.

Henderson C. Y., V. Mariotti, D. Pany-Kucera, S. Villotte y C. Wilczak. 2013. Recording Specific Entheseal Changes of Fibrocartilaginous Entheses: Initial Tests Using the Coimbra Method. International Journal of Osteoarchaeology, 23: 152-162.

Henderson C. Y., V. Mariotti, D. Pany-Kucera, S. Villotte y C. Wilczak. 2016a. The new "Coimbra method": a biologically appropriate method for recording specific features of fibrocartilaginous entheseal changes. International Journal of Osteoarchaeology, 26 (5): 925932.

Henderson, C. Y., V. Mariotti, C. Wilczak, S. Villotte y F. Santos. 2016b. The "New Coimbra Method" and the Effect of Age. Working your fingers to the bone. An interdisciplinary conference on identifying occupation from the skeleton. Coimbra, Portugal, 6-8 julio de 2016.

Henderson, C. Y., V. Mariotti, F. Santos, S. Villotte y C. Wilczak. 2017. The new Coimbra method for recording entheseal changes and the effect of age-at-death. Bulletins et Mémoires de la Société d'Anthropologie de Paris. DOI: 10.1007/s13219-017-0185-x.
Jurmain, R. 2009. Understanding "musculoskeletal stress markers": their multifactorial etiology and constraints on simplistic interpretations. Workshop in Musculoskeletal Stress Markers (MSM): limitations and achievements in the reconstruction of past activity patterns. Coimbra, Portugal, 2-3 July 2009.

Jurmain, R., S. Villotte. 2010. Terminology. Entheses in medical literature and physical anthropology: a brief review. Documentooriginadoen el Workshop in Musculoskeletal Stress Markers (MSM): limitations and achievements in the reconstruction of past activity patterns. Universidad de Coimbra, Portugal, 2 y 3 de Julio de 2009. http://www.uc.pt/en/cia/msm/MSM_ terminology3 (Última consulta: 02/02/2017).

Jurmain, R., F. Alves Cardoso, C. Henderson y S. Villotte. 2012. Bioarchaeology's Holy Grail: the reconstruction of activity. A.L. Grauer (ed) A Companion to Paleopathology, 12, 531-552, Wiley-Blackwell, Chichester.

Larsen, C. S. 2002. Bioarchaeology. Interpreting behavior from the human skeleton. Cambridge University Press. Cambridge.

Lieverse, A., V. Bazaliikii, O. Goriunova y A. Weber. 2013. Lower Limb Activity in the Cis-Baikal: Entheseal Changes Among Middle Holocene Siberian Foragers. American Journal of Physical Anthropology, 150 (3): 421-432.

Lovejoy, C. O., R. S. Meindl, T. Pryzbeck y R. P. Mensforth. 1985. Chronological metamorphosis of the auricular surface of the illium: a new method for the determination of age at death. American Journal of Physical Anthropology, 68: 15-28.

MacLaughlin, S. M. 1990. Epiphyseal fusion at the sternal end of the clavicle in a modern Portuguese skeletal sample. Antropologia Portuguesa, 8: 59-68.

Mariotti, V., F. Facchini y M. G. Belcastro. 2004. Enthesopathies - proposal of a standardized scoring method and applications. Collegium Antropologicum, 28: 145-159.

Mariotti, V., F. Facchini y M. G. Belcastro. 2007. The study of entheses: proposal of a standardised scoring method for twenty-three entheses of the postcranial skeleton. Collegium Antropologicum, 31: 291-313.

Marques, R., Silva A. M. 2006. Developmental defects detected on the vertebral colum nof a Medieval Portuguese male skeleton from the Capela de Nossa Senhora da Vitória (Porto de Mós-Leiria). XV Congreso de la Union Internationale des Sciences Préhistoriques et Protohistoriques. Lisboa, 4-9 de septiembre. 
Michopoulou, E., E. Nikita y C. Y. Henderson. 2016. A Test of the Effectiveness of the Coimbra Method in Capturing Activity-induced Entheseal Changes. International Journal of Osteoarchaeology, 27 (3): 409417.

Robb, J. 1998. The interpretation of skeletal muscle sites: a statistical approach. International Journal of Osteoarchaeology, 8: 363-377.

Santos, A. L., F. Alves-Cardoso, S. Assis y S. Villotte. 2011. The Coimbra workshop in musculoskeletal stress markers (MSM): An annotated review. Antropologia Portuguesa, 28: 135-161.

Silva, A. M. 1999a. Estudo Paleobiológico dos esqueletos exumados do Convento de São Francisco de Santarém na Campanha de 1996. 3 Relatório. Ms.

Silva, A. M. 1999b. Relatório antropológico dos restos humanos exumados do Monte da Nora (Terrugem, Elvas). Ms.

Silva. A. M., G. Carnim, J. Isidoro, M. Pinto, V. Matos, A. C. Marques. 2001. A Necrópole do século XVI instalada na villa romana do Rabaçal. M. Pessoa, L. Rodrigo, S. S. Santos, Roteiro: Rabaçal - Aldeia Cultural, 20-22,
Câmara Municipal de Penela, Penela.

Silva, A. M., S. Codinha. 2002. Antigo cemitério da Alta de Coimbra. Estudo de uma amostra óssea humana exumada junto à Rua Joaquim António de Aguiar (Coimbra). Ms.

Tereso, S. 2009. “Memória no Largo: Estudo de uma amostra osteológica humana exumada no Largo Cândido dos Reis - Santarém (XVI-XVIII)". Departamento de Antropologia, Faculdade de Ciências e Tecnologia, Universidade de Coimbra, Portugal. Tesis de Maestría. .

Ubelaker, D. 1987. Estimating age at death from immature human skeletons: an overview. Journal of Forensic Sciences, 32: 1254-1263.

Villotte, S. 2006. Connaissances médicales actuelles, cotation des enthésopathies. Nouvelle méthode. Bulletins et Mémoires de la Société d'Anthropologie de Paris, 18: 1-2.

Wilczak, C. A., V. Mariotti, D. Pany-Kucera, S. Villotte y C. Y. Henderson. 2017. Training and interobserver reliability in qualitative scoring of skeletal samples. Journal of Archaeological Science Reports, 11: 69-79. 